\title{
Multi Station Approximation and Noise Mitigation Process to OFDM Systems Using Successive JCl
}

N P SARADA Devi ( $\nabla$ saradadevi465@gmail.com ) Jawaharlal Nehru Technological University Anantapur https://orcid.org/0000-0002-6476-0362

\section{Research Article}

Keywords: OFDM process, noise analysis, approximation of station, CRLB \& throughput analysis.

Posted Date: February 28th, 2022

DOI: https://doi.org/10.21203/rs.3.rs-853784/v1

License: (9) This work is licensed under a Creative Commons Attribution 4.0 International License. Read Full License 


\section{Multi station approximation and Noise mitigation process to OFDM systems Using Successive JCI}

Mrs. N P SARADA DEVI, Prof M L RAVICHANDRA,

JNTUA, Research Scholar, ECE, Anantapur, AP, India, SRIT, HOD, ECE, Anantapur, AP, India

saradadevi465@gmail.com ravichandra.ece@srit.ac.in

Abstract-The performance of OFDM systems can be sharply deteriorated with effect of impulsive noise. It's proposed in this paper about the combined station impulse reaction approximation\& impulsive noise mitigation-process on the basis of compressed-sensing concept. From these algorithms, we can treat channel impulsive responses \& impulsive noise as joint sparse-vector. Thereafter, framework of sparse-Bayesian learning is utilised for helping estimating the stationimpulse responses, impulsive noise $\&$ data signs. Here, data sign can be known as the unknown parameter. The Cramer Rao Lower Bound's elaborated as the benchmark. From all used impulse noisemitigation techniques, proposed procedure in the paper can be used with the sub-pilot by not using past info of the station\& impulsive-noise. The model outcomes conclude at the process projected in the paper improves the efficiency in the station approximation\& enhances performance of bit error rate.
Index Terms:OFDM process, noise analysis, approximation of station, CRLB \& throughput analysis.

\section{INTRODUCTION}

A few occupations of far-off correspondence technology (example vehicular affiliations [1], quick system [2], \&narrow sea cut down affiliations [3]), transmission of information signals be truly crumbled an impulsive aggravation (IN). Wellsprings rushed upheaval novel, for instance, start clatter in automobiles [4], controls for electrical kinds of stuff [5], specific ocean rehearses [6], \& so on Diverged from added substance whiteGaussian disturbance (AWGN), careless irritation rises imprudently using brief length \& high power focal purposes. Changed repetitive division-multiplexing (OFDM) headway has-been altogether embraced generally current distant correspondence principles [7].Like manner OFDM recipients, time-space got signal has been changed over into the intermittent locale over a discrete-Fourier change (DFT), next to each sub-carrier is de- 
modulated clearly [8]. So \& so tone-bytone demodulation attains optimal most indisputable prospect ID by AWGN \& bewildering channel state info [9]. Right when this surged fuss was present, regardless, looking at repeat region disrupting impact tests shall be exceptionally reliant on, \& tone-by-tone de-modulation is in a little while absurd ever since the unconventionality of performing the joint-assertion at recipient grows un-questionably with proportion of sub-pilot [10]. Gifted rash disrupting impact cover framework plays a gigantic occupation in moving the showing of OFDM correspondence structures inside seeing added substance rash bang. Since the plentifulness of the rash uproar is consistently significantly higher than that of the establishment disturbance, it is possible to pick the presence of rushed racket by setting an edge $\&$ short period of time later to enterprise a memory less nonlinear pre-processor (e.g., cutting, blanking, or a mix there-of) to kill the effect of inspiration uproar [11]-[14]. By setting various limits, the nonlinear assessor for foolish squabble can chip away at the sign to-upheaval power degree (SNR) at their beneficiary [15]. Regardless, this systems require uproar priori appraisals to get the best edge yet persevere from execution contamination when the priori information mismatches by the time-moving upheaval experiences, which isn't that rather easy forgetting them actually furthermore. Moreover, these nonlinear pre-processor may devastate equity among OFDM subpilot, in this way happening in intercarrier impedance in the go over space [16]. Of late, there has been making interest in making stuffed recognizing (CS) based rash uproar balance frameworks that abuse the time-space scarcity of hurried upheaval [4], [6]. These frameworks all use the info of invalid qualities (i.e., tones which don't pass on data obviously pilots) of got OFDM picture for the review the IN show $\&$ thus kill it from got signal. Besides, few of them has been removed in the temperament for seeing bursty (i.e., blockspurned) rash uproar [20] by consuming created compacted perceiving speculation [12]. Notwithstanding the way that these systems show clear benefits over those based on non-linear pre-processor,standard deficiency of these evaluations is that there presentations are in general confined in proportion of invalid tones. It legitimizes rising that these methods furthermore expect that the station state information is as of now surveyed consummately before the abnormal exacerbation dispatch \& don't think about the mind-boggling impact of rash clatter on the station evaluation [13]. The obligations of this paper are as demonstrated by the going with: 
- We treat faint data pictures as hyper parameters\& foster an iterative methodology reliant upon the ExpectationMaximization (EM) evaluation for combined station evaluation, IN assessment, \& data district. Evaluation may sensibly recover the lacking flight path in any case, when these assessment cross section for the most part dull due to presence of dull data images.

- Actually not really indistinguishable from various CS engineered assessment methodologies which are used simply invalid sub-pilot, the proposed reasoning can misuse all sub-pilot for improving the IN appraisal execution. Our methodology need less invalid sub-pilot\& can drive the show up at reasonableness. Near the doubt thatstation inspiration response \& rash upheaval tests are all deficient, our proposed strategies don't necessitate other importantdata.

We pick the shut advancement Bayesian CramerRao LowerAssured (CRLB) of pilot\& rash irritation assessment.

\section{SYSTEM MODEL}

We estimate an OFDM system by Ni subpilotswhereverMisub-pilots taken for booming pilot \& $\mathrm{Ni}-$ Misub-pilots are castoff transferring data. Atfewscenarios, we ponder the system which has zerosubpilots\&these sub-pilots can also be included by firstsub-pilot with locale pilot signs as zero. At transmitter, infosigns which were swapped from causetads\& known pilot signs combined as frequencydomain OFDM $\operatorname{sign} x=\left(x_{0}, x_{1}, \gamma N-\right.$ $1)^{T}$ OFDM modulator, as inverse discreteFourier transformation (IDFT), changes frequency-domain OFDM signs in the time-domain OFDM warning sign fromwhich cyclical prefix (CP) prepended earlier saving into wireless station. Pretentious that the intersigninterferingeluded by onlydisposalof cyclicalprefaceat receiver, received time province frequency signal expressed as

$\mathrm{r}=\mathrm{HiF} * \mathrm{x}+\mathrm{u} \ldots 1$

where $\mathrm{Hi}$ is $\mathrm{Ni} \times \mathrm{Ni}$ circulant surrounding substance whose prior pier will be formed zero-padded passage impulse retort vector $h=\left(h_{0}, h \ldots \ldots \ldots \ldots . . . . h L-1\right)^{T}, \mathrm{~L}$ is distance impulse signalstation data. $F$ will be Unitary Ni-Point- discrete Fourier transform (DFT) vector $(m, n)$ elements are conjugate transpose.

$y=F_{v}=F H F^{*} x+F^{i}+F g=\Delta x+F_{1}^{l}+n$.

where $\Delta x$ is diagnonal vector matrix with help of frequency response station ,h is diagnoanl vector matrix elements, finally DFT is impulse vector station weight matrix data through which it is still a station of AWGN for $\mathrm{F}$ is unitary matrix data. 
$p(v)=\prod_{i=1}^{N} \sum_{k=1}^{k} \pi k f_{k}\left(\begin{array}{c}1 \\ v_{1}\end{array}\right) \ldots 3$

Where $f_{k}$ denotes a complex Gaussian data analysis for wireless communication. $\Delta k$, $\pi k$ is the mixing probability of impulse noise pilot's are expected to be autonomous \& identically circulated.

\section{PROPOSED APPROACHES}

From equation (2), received OFDM signshould statistically characterized as

$$
\begin{gathered}
\boldsymbol{y}=\operatorname{diag}(\tilde{h}) \boldsymbol{x}+\boldsymbol{F i}+\boldsymbol{n} \\
=\operatorname{diag}(\tilde{h}) \boldsymbol{x}+\boldsymbol{F i}+\boldsymbol{n} \\
\sqrt{n x} F_{L} H+\boldsymbol{F i}+\boldsymbol{n} \quad \ldots .4
\end{gathered}
$$

where $\mathrm{X}$ is diag with diagonal vector with high occurrence stream sign is $\mathrm{x}$ is crosswise element vector.Free space wireless communication discrete time frequency impulse response (CIR) is $\mathrm{L}$ for length \& S for solvable propagation paths can me modelled as

$h_{1}=\sum_{s=1}^{s} \alpha_{\varsigma} \delta\left[1-\tau_{s}\right]_{1} 0 \leq l \leq L-1 . .5$

Where $\alpha_{\varsigma} \& \tau_{s}$ indicate path improvement\& the regulated path delay $s$-th path, correspondingly. Without loss of a simplification, undertake $0 \leq \tau_{0} \leq \tau_{1} \leq$ $\tau_{s-1} \leq \mathrm{L}-1$. Providentially, several theoretical examination\&investigational outcomes has difference in those wireless networks are sparse environment, i.e., in the CIR model (5), measurements of the CIR $L$ may be vast, but count of lively paths $S$ with Significantgains usually tiny, i.e., $S<<L$, particularlyin wireless wide band communications

$y=\phi \omega+n \quad 6$

where matrix $\phi$ is clearly an undetermined matrix. Provisionally noting that the both IN vector $\boldsymbol{I} \&$ CIR vector $\boldsymbol{h}$ are bare, the differentcreated vector $\boldsymbol{w}$ also viewed by bare vector sensibly. So approximation of $\boldsymbol{w}$ in (6) can measured as archetypalcrushedidentifyingtricky.

Moreover, note that info signs, namely fewportionof matrix $\phi$, is unfamiliar at the receiver \& need to the measured, which demands the advance of methods that the capable of control partially unidentifiedlexicon matrices.

Algorithm : SJCI

Input: $y_{P^{\prime}} \phi_{P}, \boldsymbol{r} \max$, and $\varepsilon$

Output: $\widehat{w}$

Given prior vector value: $\Gamma(0)=$ I and $\lambda(0)=1$

While $\left\|\mu(r+1)-\mu^{(r)}\right\|_{2}^{2} \geq \varepsilon$ and $\gamma \leq$ $\gamma \max d o$

Loop Step:

Update $\mu, \Gamma$ based on vector optimization

Update $\Sigma, \lambda$ based on Weight matrix data

end Loop

return $\widehat{w}=\boldsymbol{\mu}$ 
The recital of beyond proposed SJCI is limited by count of priorsubpilot. However, raising the count initial subpilot will lead reducing spectra efficiency\& system output. If info ofdata subpilot of OFDMsign be explored, it is desired to progress the approximation performance of station\& IN with system output guarantee. $\left.\boldsymbol{\mu} \Delta\right|_{\mu_{i}^{w}} ^{\mu_{j}}=\frac{1}{\lambda} \Sigma \phi^{*} y \quad 7$

$\Sigma_{-}^{\Delta}\left(\begin{array}{cc}\Sigma_{j j} & \Sigma_{j i} \\ \Sigma_{i j} & \Sigma_{i i}\end{array}\right)=\Gamma-\Gamma \phi^{*}\left(\lambda_{1}+\phi \Gamma^{*}\right)^{-1} \phi \Gamma \quad 8$

Where $\boldsymbol{\mu}_{\boldsymbol{j}} \& \boldsymbol{\mu}_{\boldsymbol{i}}$ are subsetsequivalent to station\& IN blocks in the mean vector $\boldsymbol{\mu}$ correspondingly\&same process is applied to covariance matrix $\boldsymbol{\Sigma}$.

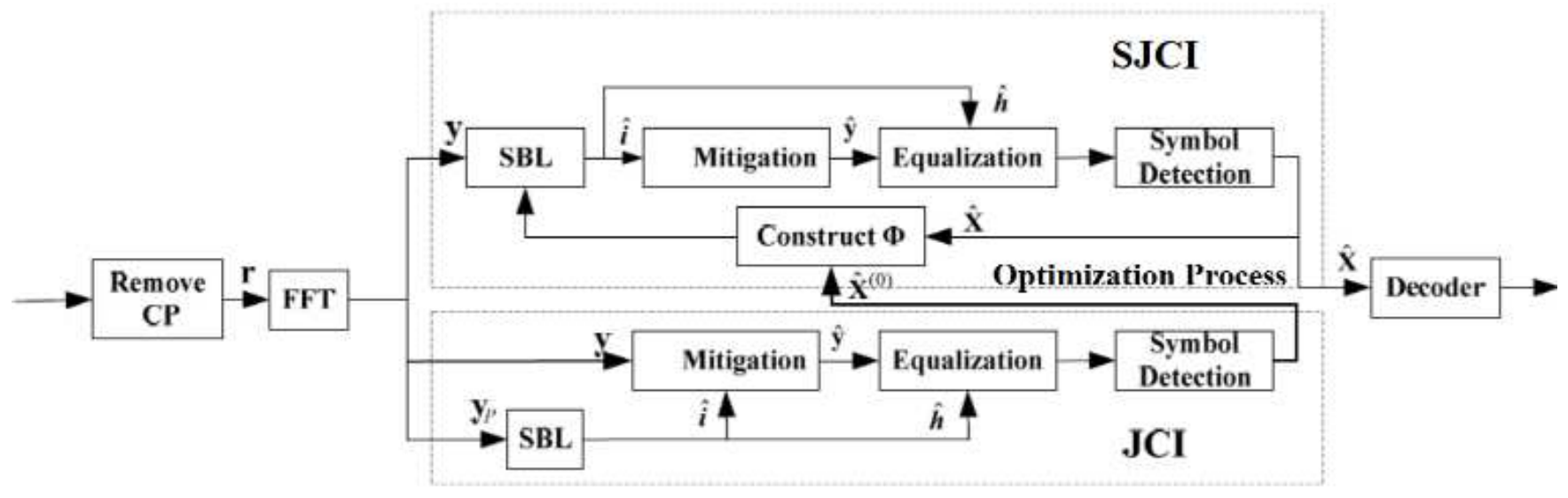

Figure 1. Block diagram of our proposed Methodology.

CRLB Analysis: The CRLB deliversanultimate lower limit on the MSE performance of unbiased estimators. For random parameter approximation with availability of aimportantinformation, the Cramer-Rao Lower Bound (CRLB) is currently used to obtain fewer bounds of dynamical Rayleigh station complex gains approximation in OFDM system. CRLB for SBL algorithm was derived for our proposed SJCI algorithm. $\operatorname{CRLB}(\mathbf{w})=$ $\left(J^{\omega}\right)^{-1}$

$$
\begin{gathered}
\left(\frac{\phi^{*} \phi}{\lambda}+\Gamma^{-1}\right)^{-1} \\
\Gamma-\Gamma \phi^{*}\left(\lambda_{1}+\phi \Gamma \phi^{*}\right)^{-1} \phi \Gamma \sum w_{x, y}
\end{gathered}
$$

The output of our projectedplanning derived in observed that SJCI \& JCI can gain higher output than the other algorithms. By manipulating all tones to estimate the station\& impulsive noise, SJCI can achieve higher throughput performance. The proposed algorithm is greater count user's data transmission at a time to transmit both the transmission \& reception vice-versa.

IV. EXPERIMENTAL RESULTS 


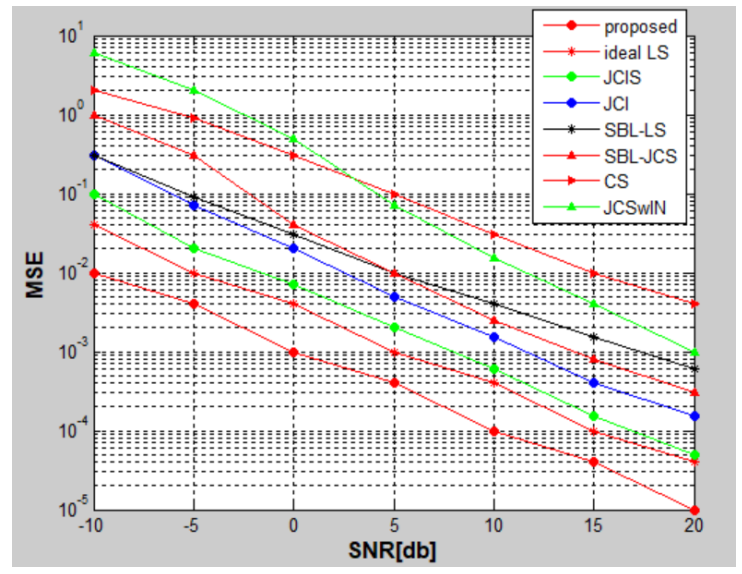

Figure 2. The MSE station approximation versus SNR. The countby pilot subpilot is 56. The count of MIMO multisubpilot is 80.

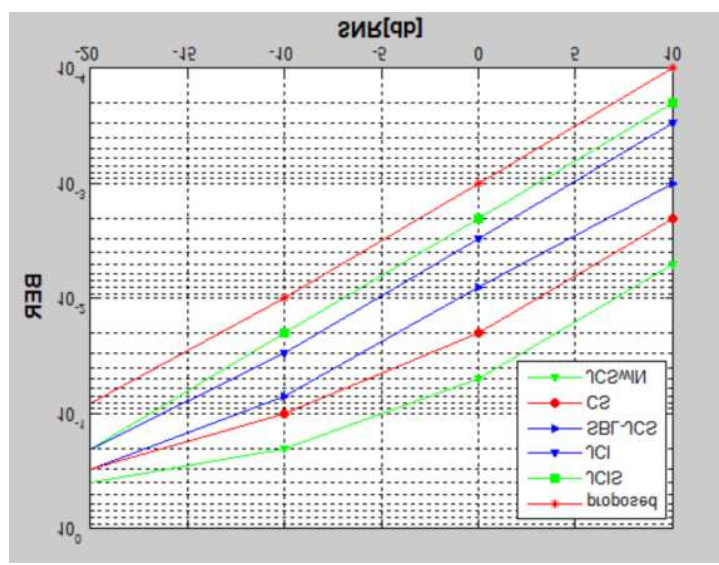

Figure 3. BER versus SNR in uncoded OFDM system. The count of pilot subpilot is 56. The count of MIMO multisubpilot is 80 .

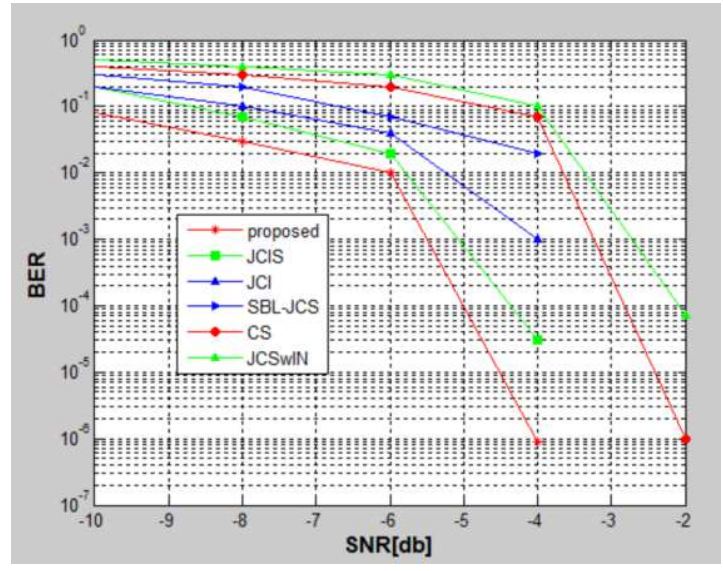

Figure 4. BER versus SNR in coded OFDM system. The countpriorsub-pilot56. The count of MIMO multisubpilot80.

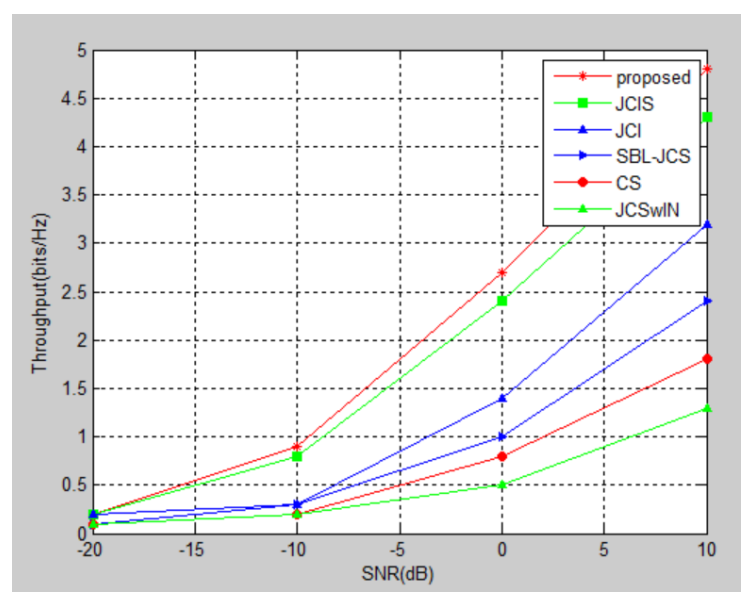

Figure 5. System throughput versus SNR.

The count pilot sub-pilot 64. The countofMIMO multisub-pilot is 80 .

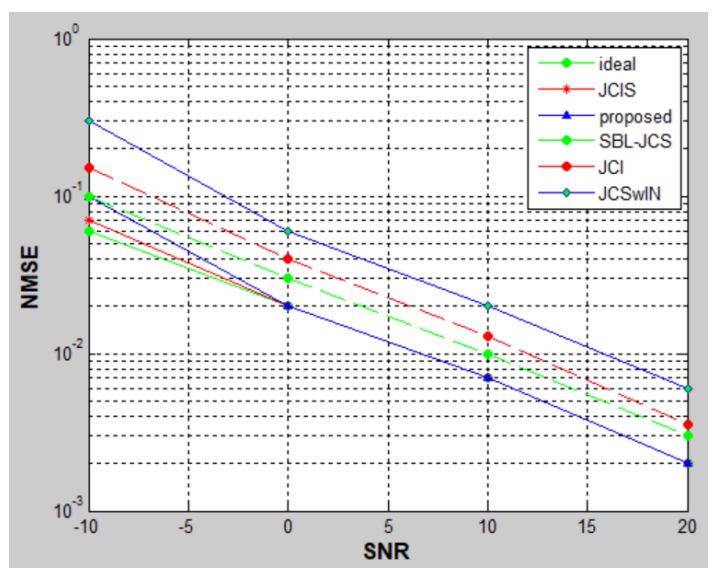


Figure 6. The MSE performance contrast between JCI \&SucceedingJCI with various count of pilot tones.

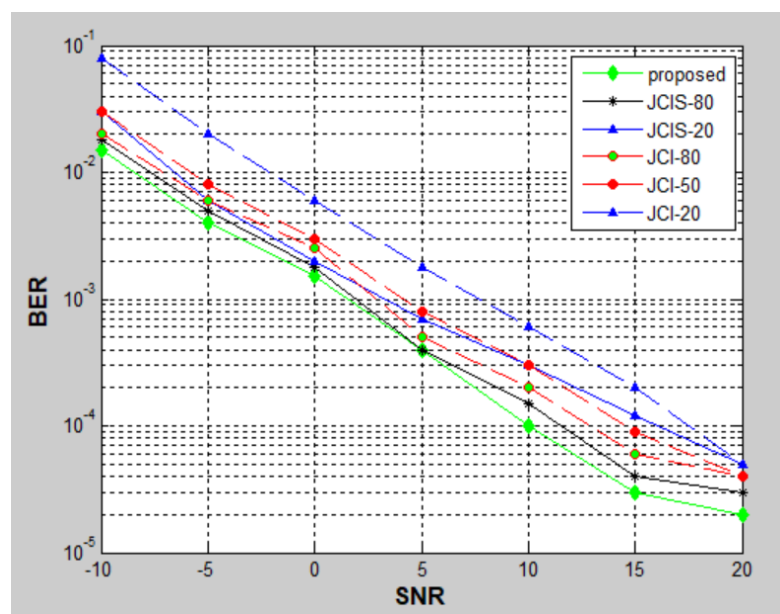

Figure7. The uncoded BER action contrast among JCI \& Successive JCI different count of zero tones.

\section{CONCLUSION}

For the execution of combine sparse stationapproximation, impulsive noise mitigation, information detection is utilised by OFDM systems. By considering stationscarcity\& impulsive noise by that time zone, we implement wide sparse vector for the representation of the impulsive noise \&stationorganized. For the approximation of vector, we proposed JCI's procedure to give better efficiency in stationapproximation\& impulsive station cancellation applying the data detection respectively. The analytical expression of derived for Successive JCI algorithm. The proposed scheme main aim of work is a greatercount of user's data transmission at a time with minimum time delay \&MSE performance was deteriorated with the conventional techniques \& closed at the lower bound. The results show that the simulation techniques are efficient at BER performance with less pilots \& null subpilot\& offers improvements in spectral efficiency.

\section{DECLARATIONS}

Funding:Funding information is not applicable / No funding was received.

\section{Conflicts of interest/Competing} interests: Not applicable

Availability of data and material:All data generated or analysed during this study are included in this published article (and its supplementary information files).

Code availability:software application

Authors' contributions: Not applicable

\section{REFERENCES}

[1] S. F. Hasan, X. Ding, N. H. Siddique, \& S. Chakraborty, "Measuring disruption vehicular communications," IEEE Trans. Veh. Technol., vol. 60, no. 1, pp. 148-159, Jan. 2011.

[2] S. W. Lai \& G. G. Messier, "Using the wireless \& PLC stations for diversity," IEEE Trans. Commun., vol. 60 , no. 12 , pp. $3865-$ 3875, Dec. 2012.

[3] Z. Wang, S. Zhou, J. Catipovic, \& P. Willett, "Asynchronous multiuser reception 
for OFDM in undergroundwater acoustic communications," IEEE Trans. Wireless Commun., vol. 12, no. 3, pp. 1050-1061, Mar. 2013.

[4] S. Liu, F. Yang, W. Ding, \& J. Song, "Double kill: Compressivesensing-based narrow-b\&intervention\& impulsive noise mitigation for vehicular communications,", IEEE Trans. Veh. Technol., vol. 65, no. 7, pp. 5099-5109, Jul. 2016.

[5] F. Sacuto, F. Labeau, \& B. L. Agba, "Wide b\& time-correlated model for wireless communications behind impulsive noise with the power substation,' IEEE Trans. Wireless Commun., vol. 13, no. 3, pp. 1449-1461, Mar. 2014.

[6] X. Kuai, H. Sun, S. Zhou, \& E. Cheng, "Impulsive noise mitigation in underwater acoustic OFDM systems," IEEE Trans. Veh. Technol., no. 65, no. 10, pp. 8190-8202, Oct. 2016.

[7] IEEE St\&ard for Information technologyLocal \& metropolitan area systems-Specific requirements-Part 11: Wireless LAN Medium Access Control (MAC) \& Physical Layer (PHY) ConditionsAdjustment 6:Wireless Access in Vehicular Environments, IEEE St\&ard 802.11p Std., Jul. 2010.

[8] D. Tse\& P. Viswanath, Fundamentals of Wireless Communication. Cambridge, U.K.: Cambridge Univ. Press, 2005.

[9] Y. Liu, Z. Tan, H. Hu, L. J. Cimini, \& G. Y. Li, "Stationapproximation for OFDM,"
IEEE Commun. Surveys Tuts., vol. 16, no. 4, pp. 1891-1908, 4th Quart., 2014.

[10] A. Mahmood, M. Chitre, \& M. A. Arm\&, "Detecting OFDM signals in alpha-stable noise," IEEE Trans. Commun., vol. 62, no. 10, pp. 3571-3583, Oct. 2014.

[11] S. V. Zhidkov, "Performance analysis \& optimization OFDM receiver through blanking nonlinearity by impulsive noise environment," IEEE Trans. Veh. Technol., vol. 55, no. 1, pp. 234-242, Jan. 2006.

[12] S. V. Zhidkov, "Analysis \& comparison of count ofmodest impulse noise mitigation schemes for OFDM receivers," IEEE Trans. Commun., vol. 56, no. 1, pp. 5-9, Jan. 2008.

[13] U. Epple\& M. Schnell, “Advanced blanking nonlinearity for mitigating impulsive interference in OFDM systems,' IEEE Trans. Veh. Technol., vol. 66, no. 1, pp. 146-158, Jan. 2017.

[14] F. H. Juwono, Q. Guo, Y. Chen, L. Xu, D. D. Huang, \& K. P. Wong, "Linear combining of nonlinear preprocessors for OFDM-based powerline communications," IEEE Trans. Smart Grid, vol. 7, no. 1, pp. 253-260, Jan. 2016.

[15] N. Rožić, P. Banelli, D. Begušić, \& J. Radić, "Multiple-threshold estimators for impulsive noise suppression in multicarrier communications," IEEE Trans. Signal Process., vol. 66, no. 6, pp. 1619-1633, Mar. 2018.

[16] D. Darsena, G. Gelli, F. Melito, \& F. Verde, "ICI-free equalization in OFDM 
systems with blanking preprocessing at receiver by impulsive noise mitigation," IEEE Signal Process. Lett., vol. 22, no. 9, pp. 13211325, Sep. 2015.

[17] G. Caire, T. Y. Al-Naffouri, \& A. K. Narayanan, "Impulse noise cancellation in OFDM: An application of compressed sensing," in Proc. IEEE Int. Symp. Inf. Theory, Toronto, ON, Canada, Jul. 2008, pp. 1293-1297.

[18] J. Lin, M. Nassar, \& B. L. Evans, "Impulsive noise mitigation in powerline communications using sparse Bayesian learning," IEEE J. Sel. Areas Commun., vol. 31, no. 7, pp. 1172-1183, Jul. 2013.

[19] T. Y. Al-Naffouri, A. A. Quadeer, \& G. Caire, "Impulse noise approximation\& removal for OFDM systems,' IEEE Trans. Commun., vol. 62, no. 3, pp. 976-989, Mar. 2014.

[20] L. Lampe, "Bursty impulse noise detection by compressed sensing," in Proc. IEEE Int. Symp. Power Line Commun. Appl., Udine, Italy, Apr. 2011, pp. 29-34

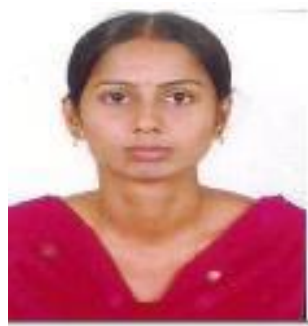

Mrs. N P SARADA DEVI

received B.Tech and M.Tech degrees from JNTU anantapur, Andhra Pradesh in 2009 and 2011 respectively. Now working as lecturer in SKU college of engineering and technology, anantapur. Presently pursuing $\mathrm{PhD}$ at JNTUA.

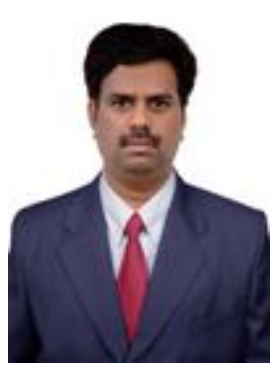

Prof. M L RAVI CHANDRA received M.tech from JNTUA and $\mathrm{PhD}$ from JNTUH. He has several years of teaching experience and now he was the HOD of ECE department at SRIT, Anantapur. 